\title{
An open-label forearm-controlled pilot study to assess the effect of a proprietary emollient formulation on objective parameters of skin function of eczema-prone individuals over 14 days
}

This article was published in the following Dove Press journal:

Clinical, Cosmetic and Investigational Dermatology

27 July 2017

Number of times this article has been viewed

\section{Michael Paul Wakeman}

Department of Cancer Sciences, School of Medicine, University of Birmingham, Edgbaston, Birmingham, UK
Correspondence: Michael Paul Wakeman Department of Cancer Sciences, School of Medicine, University of Birmingham, Edgbaston, Birmingham BTI5 2TT, UK Email mikepwakeman@gmail.com
Background: This study examines the efficacy of a new plant-based emollient and assesses product acceptability.

Methods: Primary efficacy endpoints were improvement in transepidermal water loss, hydration, skin elasticity and firmness, erythema, and skin roughness and smoothness as measured using the versions of Tewameter, Corneometer, Cutometer, Mexameter, and Visioscan VC98, respectively. The cream was applied twice daily by 32 participants to an area of one forearm unaffected by eczema, while the same area of the other forearm was used as a control. Measurements were taken at day 0 and day 14 . Secondary endpoints assessed the acceptability of the product. Results: At the end of 2 weeks, transepidermal water loss, hydration, skin elasticity and firmness, erythema, and skin roughness and smoothness improved. All changes were statistically significant $(p<0.01)$. The rate of satisfaction with the emollient properties was $82 \%$, and the rate of absorption into the skin was $88 \%$. Results show that the emollient hydrates and repairs eczema-prone skin with high levels of acceptability.

Keywords: eczema, Suvex, moisturizer, emollient, hydration, skin barrier function

\section{Introduction}

Eczema is characteristic of those conditions with dry, scaly skin that are typically treated with emollients and topical steroids. Effective emollients are able to soften and moisturize the skin, thereby repairing the epidermal barrier at the level of the stratum corneum and as a result often allowing the otherwise use of topical steroids to be reduced either in frequency or in potency. ${ }^{1}$ Apart from preventing possible environmental triggers from penetrating the skin, they may also additionally deliver an intrinsic anti-inflammatory action and provide antipruritic benefits., ${ }^{2,3}$

Indeed, clinical guidelines relating to the treatment of conditions such as atopic eczema often recommend that emollients be used as the foundation of management, especially in children, to moisturize the skin throughout the day and night, and as substitutes for soap. ${ }^{4}$ Essentially, emollients produce their effect either by forming a film over the skin that prevents water from evaporating from the skin or by a humectant action that attracts water into the skin. ${ }^{5-7}$ Occlusive compounds such as liquid and white soft paraffin are typical in the former category, while the latter often contain compounds such as glycerine and urea. Traditional emollients act to seal the gaps that occur between desquamated corneocytes to form a less rough surface, to flatten and provide cohesion to their curled edges. ${ }^{8}$ 
Given that eczema is such a significant problem globally, research continues on an ongoing basis to identify an ideal emollient. However, little evidence exists to establish the benefit of one emollient compared with another. ${ }^{9}$ From a clinical perspective, compliance and the personal preference of the patient appear to be significant factors in determining the choice of the product. In general, most clinicians and patients prefer a fragrance and dye-free emollient with a daily application regimen twice or thrice.

The choice of emollient depends on a number of factors, such as patient preference, the site to which it is to be applied, and the severity of the condition. However, while the thicker and more greasy ointments, such as those based on white soft paraffin are effective emollients, because of poor absorption into the skin and the resulting greasy film they leave on it, many patients object to their use, which leads to poor compliance on a routine basis. ${ }^{10,11}$ As a result, many patients prefer emollients that are oil-in-water emulsions. Although these are cosmetically more appealing and better absorbed into the skin, they have a reduced occlusive effect and tend to be more easily removed onto clothing due to their reduced oil content, thus leading to a need for more frequent application. ${ }^{12}$

There is now a growing amount of clinical and experimental evidence to indicate that the skin should be considered not only as the target in atopic eczema but also as the starting point of the atopic march. ${ }^{13}$ If the skin is to function effectively, the skin barrier needs to be healthy. Essentially, this means that water needs to be retained to keep the skin hydrated and comfortable, and it also needs to operate as an intact barrier capable of repelling potential toxins and irritants. Should the skin become irritated or extremely dry, micro fissures develop in the stratum corneum, which result in dry, rough, and scaly skin. When the barrier is broken down in this way, it becomes difficult to maintain adequate hydration of the cells, and hence, their ability to repel pathogens and toxins also becomes compromised. ${ }^{14-19}$

Several topical agents have now been approved as medical devices to assist in restoring barrier function. Some of these include hyaluronic acid and glycyrrhetinic acid (a derivative of liquorice), which, along with other components, impart antioxidant, moisturizing, and anti-inflammatory properties. Other formulations have been developed to improve barrier function by delivering a particular ratio of ceramides, cholesterol, and fatty acids to the skin surface, which augments the skin's structural defense. ${ }^{20,21}$ In addition, lipids, which moisturize the skin and mimic the stratum corneum framework, contribute to a dampened inflammatory response.
Desirable characteristics for such an approach is to provide $^{22}$

- Replenishing qualities to provide protection, with moisturizing and conditioning properties;

- A fine protective filmic coating that provides everyday defense against loss of moisture and everyday irritants;

- A unique combination of essential fatty acids, proteins, and other compounds such as polyphenols and polysaccharides combined in such a way as to maintain the integrity of the water-lipid film and penetrate into the deeper layers of the skin to form an effective protective barrier that holds moisture in the skin; ${ }^{15}$

- Natural plant ingredients that actively reduce the erythema and itch accompanying dry skin and so breaking the itch/scratch cycle which is thought to be a key objective in terms of helping skin heal effectively. ${ }^{16}$

Restoring the normal $\mathrm{pH}$ in skin conditions is also important since imbalances, especially in the essential fatty acid matrix in the stratum corneum, appear to compromise the barrier capabilities of the skin, which facilitates the initiation of allergenic and inflammatory triggers and irritants and increases the rate of water loss. As the skin becomes drier, this can exacerbate the situation. Hence, intense moisturization is important. Furthermore, it is now appreciated that the skin barrier function of those patients experiencing conditions where dry skin is troublesome is impaired not only in the symptomatic areas but also in those parts of the skin that appear to have a normal appearance..$^{23}$

Some necessary elements of a topical emollient treatment capable of overcoming epidermal barrier dysfunction are as follows: a steroid-free petroleum, lanolin, and fragrance-free product, providing a solution to dry, scaly, and itchy skin and delivering ingredients that not only reestablish the essential fatty acid balance of the skin but also provide a soothing effect in a formulation that has the feel, texture, and performance of a top-class emollient.

The proprietary formulation under investigation has been developed with all of the above considerations in mind, and this work seeks to establish whether it performs well as an emollient preparation in individuals who have a history of atopic eczema.

\section{Materials and methods}

This study was an open-label assessment of Suvex Soothe emollient cream (Naturalife Ltd, Rathnew, Ireland). It contains ethically sourced essential fatty acid rich oils, such as shea and cupuaçu butters, and rosehip and Mediterranean 
olive oil that also deliver anti-inflammatory polyphenolicand phytosterol-based compounds along with aloe vera, and two branded plant extract products, Defensil and HomeoSoothe, as well as vitamin E. It is fragrance free. The study population consisted of 40 male and female individuals aged 20-72 years with a history of eczema and dry skin. A full ingredient list appears in Supplementary materials. All participants provided witnessed informed consent. Exclusion criteria included significant concurrent skin disease affecting the area to be assessed; history of allergy to the test product ingredients, such as aloe vera; concurrent administration of systemic or topical medications that might impact upon skin function; and any visible abnormalities of the evaluated area of skin, such as the presence of hair, tattoos, birthmarks, scaring, or skin irritation that might affect the measurements being undertaken. The test product was a white cream, presented in a $100 \mathrm{~mL}$ tube delivering approximately one fingertip unit - about $1 \mathrm{~mL}$ per squeeze.

Participants were recruited via posters in pharmacies that have been accredited by the Royal Pharmaceutical Society of Great Britain to perform scientific research, and the study was approved by the Leicester Central Ethics Committee. When participants arrived at the study site, written informed consent was obtained for this study while they rested, and they rolled up their sleeves which allowed their skin to acclimatize to the environment. This was kept at $61 \%-65 \%$ relative humidity and $21^{\circ} \mathrm{C}-22^{\circ} \mathrm{C}$ for all assessments. An area of skin, each measuring $5 \times 4 \mathrm{~cm}$, was identified just below the elbow on the inner side of each forearm. Participants were instructed to apply the dose of emollient to this area of right forearm, while the corresponding area of the left forearm was used as a control. The intervention consisted of the application of a single fingertip unit of Suvex massaged gently into a designated area of the right forearm twice daily for 14 consecutive days, thereby mimicking normal clinical recommendations. The tubes were weighed at the beginning and the end of the study to ascertain the quantity of the emollients that had actually been used.

On day 1 and on day 14, the participants visited the study site for Corneometer, Tewameter, Mexameter, Cutometer, and Visioscan measurements of the designated areas of their skin. These measurements were made for each forearm before the first application (these day 1 measurements were considered "baseline" scores). Final measurements were repeated on day 14. At least 6 hours had elapsed since the last application of the emollient before the day 14 readings were made. No visits to the test sites otherwise occurred. Throughout the trial, participants recorded their assessments of their subjective experience of using Suvex in a satisfaction questionnaire. It was requested that participants refrain from washing, showering, or bathing their forearms on skin assessment days, but were allowed to do so whenever and as often as usual on intervening days. However, they were instructed not to do this for 2 hours after each application. In this way, it was hoped to replicate normal washing, showering, or bathing throughout the duration of the trial. Subjects were excluded from using any other emollient preparation on their forearms during the study. Similarly, any other systemic or topical treatments considered likely to interfere with the study outcome by the chief investigator were prohibited, unless deemed necessary for the well-being of a participant by a healthcare professional. In the event that this might happen, that participant was to be subsequently excluded from the day 14 assessment. The use of any other concurrent medication by participants throughout the study was recorded. For all of the assessments discussed below, all measurements reported are the mean of three assessments obtained at the selected sites on a participant's skin on day 0 and day 14. Data are presented as mean and SD. Results of every data point recorded were compared using Student's $t$-test for numerical values of each parameter. All comparisons were made two tailed, and $p$ values of $<0.05$ were considered statistically significant.

\section{Objective skin function measurements \\ Corneometer MPA 5}

A Corneometer CM825 probe with a Multiprobe Adapter MPA5 (Courage and Khazaka, Koln, Germany) was used to preform noninvasive assessments of the level of hydration of the participant's stratum corneum. It delivers validated and well-confirmed measurements of hydration levels of the skin. ${ }^{24,25}$ The corneometer scores are presented in arbitrary units. As with all assessments discussed below, all measurements reported here are the mean of three assessments obtained at the selected sites on a participant's skin.

\section{Cutometer MPA5}

A Cutometer (Courage \& Khazaka Electronic GmbH, Cologne, Germany) was used to evaluate parameters relating to skin elasticity. It uses a controlled vacuum applied through a circular aperture to measure the vertical deformation occurring in the skin under this force. ${ }^{26}$ This time/ strain methodology is applied over a 5-second application of suction followed by a 3 -second phase of relaxation, repeated over 3 consecutive cycles. A 2-mm diameter probe was used and a constant suction of 500 mbar applied. The curves of values obtained were analyzed using the MPA 580 software, 
and two parameters - R0, a measure of skin firmness, and $\mathrm{R} 2$, the overall elasticity of the skin - were determined.

\section{Mexameter MPA5}

The Mexameter measures the intensity of melanin pigmentation and erythema using reflectance spectrophotometry. The literature indicates that the instrument delivers a high degree of discrimination and sensitivity to detect small changes in erythema and pigmentation, with satisfactory reproducibility. ${ }^{27}$

\section{Tewameter MPA 5}

Transepidermal water loss (TEWL) assesses water evaporation through the skin and hence provides an insight into the integrity of the barrier of the skin. ${ }^{28}$ It is measured in $\mathrm{g} / \mathrm{m}^{2} / \mathrm{h}$. It is a development of Nilson's vapor pressuregradient method of estimation. It was measured using a Tewameter MPA5 (Courage-Khazaka Electronic, Cologne, Germany)

\section{Visioscan ${ }^{\circledR}$ VC98}

Visioscan VC98 (Courage and Kazaka Electronic GmbH Cologne, Germany) uses special illumination and evaluation technology to provide a graphic image of the skin defined in four clinical parameters: skin roughness-Ser, skin smoothness-SEsm, skin scaliness-SEsc, and skin wrinkles-Sew. These parameters correspond to the condition of the skin surface. The device uses a camera and UV-A light video to capture high-resolution images. For the purposes of this trial, only Ser and SEsm were relevant. ${ }^{29}$

\section{Assessment of user satisfaction}

A questionnaire was designed to determine participants' level of satisfaction that Suvex delivered as an emollient, its effectiveness as an emollient, how well it absorbed into the skin, and the efficacy of the product as an emollient using a 5-point Likert scoring system, where $1=$ strongly dislike/disagree, $2=$ slightly dislike/disagree, $3=$ neither dislike/disagree nor like/agree, $4=$ =slightly like/agree, and 5=strongly like/agree.

Participants were also asked to assess the degree to which the emollient has a pleasant smell and consistency, how likely they would use it again, if the product was available on prescription, and whether they would request their health professional to prescribe it. Details of the questionnaire appear in Figure S1. In order to establish a mean score for an individual question, each score was multiplied by the frequency at which it occurred, and this number was divided by the total number of responses for that question.

\section{Results}

Forty screened participants enrolled for the study and 32 completed it. Reasons for failing to return for follow-up were given as vacation time, inconvenience, and other domestic issues rather than any problems with the trial cream. Of those completing the trial, 12 were men aged between 30 and 59 years, and 20 were women aged between 20 and 72 years. Twelve subjects reported the use of a concomitant medicine. In each case, these were oral treatments and consisted of interventions such as oral contraceptives or medications for cardiovascular disease. Since these participants had been using these medications for some time prior to the intervention, it was considered unlikely that they would impact upon the outcome of this study. The evaluation of the quantity of emollients used by all participants was generally within the range anticipated as a result of applying the recommended dosage.

\section{Efficacy of evaluations}

\section{Transepidermal water loss (TEWL)}

Table 1 summarizes the results relating to this parameter. At baseline (D0), the mean TEWL was 11.74 \pm 3.11 $\mathrm{gm}^{2} / \mathrm{h}$ (mean $\left.\pm \mathrm{SD}\right)$, and at D14, the TEWL was reduced to $10.47 \pm 2.52 \mathrm{~g} / \mathrm{m}^{2} / \mathrm{h}$ (mean $\left.\pm \mathrm{SD}\right)$. The change for TEWL was $12 \%(p<0.01)$ by paired $t$-test. Figure 1 illustrates these changes graphically.

\section{Hydration}

Table 2 summarizes the results relating to this parameter. At baseline (D0), the mean hydration was $36.45 \mathrm{AU} \pm 10.51$

Table I Transepidermal water loss at baseline (day 0 ) and end of trial (day 14)

\begin{tabular}{|c|c|c|c|c|c|c|c|}
\hline \multicolumn{8}{|c|}{ Transepidermal water loss $\left(\mathrm{g} / \mathrm{m}^{2} / \mathrm{h}\right)$} \\
\hline & Time & $\mathbf{N}$ & Median & Mean & SD & Min & $\operatorname{Max}$ \\
\hline \multirow[t]{2}{*}{ Suvex } & OD & 32 & 10.9 & 11.74 & 3.11 & 8.1 & 20.4 \\
\hline & I4D & 32 & 9.9 & 10.34 & 2.52 & 7.2 & 18.3 \\
\hline \multirow[t]{2}{*}{ Control } & OD & 32 & 10.8 & 11.76 & 3.14 & 8.0 & 20.1 \\
\hline & I4D & 32 & 10.7 & 11.77 & 3.13 & 8.1 & 20.2 \\
\hline
\end{tabular}

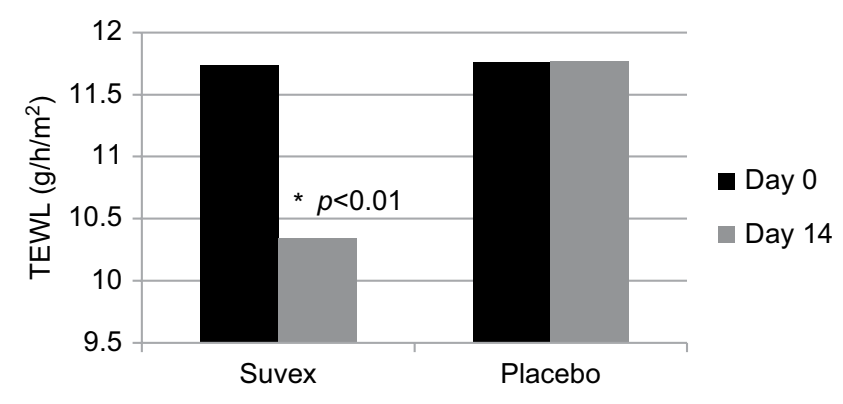

Figure I Mean transepidermal water loss (TEWL) at baseline (day 0 ) and end of trial (day 14). 
(mean \pm SD), and at D14, the hydration had increased to 42.55 AU \pm 12.7 (mean $\pm \mathrm{SD}$ ). There was a significant change in hydration from day 0 to day 14 , and it was $16.7 \%(p<0.01)$ by paired $t$-test. Figure 2 illustrates these changes graphically.

\section{Elasticity}

Table 3 summarizes the results relating to this parameter. At baseline (D0), the mean elasticity was $0.810 \mathrm{AU} \pm 0.04$ (mean \pm SD), and at D14, the elasticity had increased to 0.920 $\mathrm{AU} \pm 0.0 .05$ (mean $\pm \mathrm{SD}$ ). The elasticity changed significantly by $13.5 \%(p<0.01)$ by paired $t$-test. Figure 3 illustrates these changes graphically.

Table 2 Hydration at baseline (day 0) and end of trial (day 14)

\begin{tabular}{|c|c|c|c|c|c|c|c|}
\hline \multicolumn{8}{|c|}{ Hydration (AU) } \\
\hline & Time & $\mathbf{N}$ & Median & Mean & SD & Min & Max \\
\hline \multirow{2}{*}{ Suvex } & OD & 32 & 34.90 & 36.45 & 10.51 & 17.3 & 56.0 \\
\hline & I4D & 32 & 41.72 & 42.55 & 12.70 & 20.0 & 60.9 \\
\hline \multirow[t]{2}{*}{ Control } & OD & 32 & 34.10 & 36.39 & 10.39 & I7.I & 55.7 \\
\hline & I4D & 32 & 34.03 & 36.41 & 10.41 & 17.3 & 55.5 \\
\hline
\end{tabular}

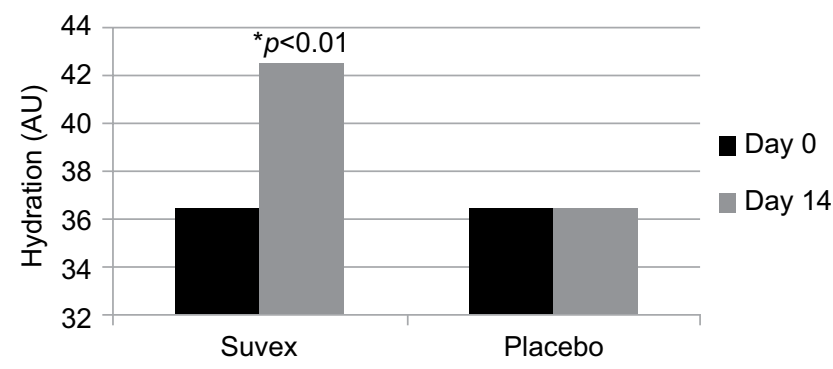

Figure 2 Mean hydration at baseline (day 0 ) and end of trial (day 14).

Table 3 Elasticity at baseline (day 0 ) and end of trial (day 14)

\begin{tabular}{|c|c|c|c|c|c|c|c|}
\hline \multicolumn{8}{|c|}{ Elasticity (AU) } \\
\hline & Time & $\mathbf{N}$ & Median & Mean & SD & Min & Max \\
\hline \multirow[t]{2}{*}{ Suvex } & OD & 32 & 0.826 & 0.810 & 0.04 & 0.670 & 0.826 \\
\hline & I4D & 32 & 0.931 & 0.920 & 0.05 & 0.765 & 0.931 \\
\hline \multirow[t]{2}{*}{ Control } & OD & 32 & 0.828 & 0.811 & 0.04 & 0.672 & 0.819 \\
\hline & I4D & 32 & 0.825 & 0.813 & 0.04 & 0.674 & 0.823 \\
\hline
\end{tabular}

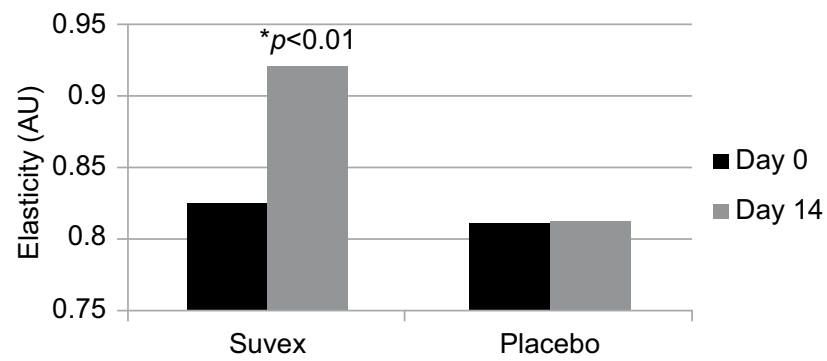

Figure 3 Mean elasticity at baseline (day 0 ) and end of trial (day 14).

\section{Firmness}

Table 4 summarizes the results relating to this parameter. At baseline (D0), the mean firmness was 0.264 AU \pm 0.071 (mean $\pm \mathrm{SD}$ ), and at D14, the firmness had reduced to 0.226 $\mathrm{AU} \pm 0.075$ (mean $\pm \mathrm{SD}$ ). The firmness changed significantly by $14.3 \%(p<0.01)$ by paired $t$-test. Figure 4 illustrates these changes graphically.

\section{Erythema}

Table 5 summarizes the results relating to this parameter. At baseline (D0), the erythema level was 321.8 AU \pm 79.5 (mean $\pm \mathrm{SD}$ ), and at D14, the level had changed to 292.2 AU \pm 76.5 (mean $\pm S D$ ). The significant percentage change for melanin/redness was $10.1 \%(p<0.01)$ by paired $t$-test. Figure 5 illustrates these changes graphically.

\section{Roughness}

Table 6 summarizes the results relating to this parameter. At baseline (D0), the mean roughness was 2.25 $\mathrm{AU} \pm 1.31$ (mean $\pm \mathrm{SD}$ ), and at D14, it was $2.55 \mathrm{AU} \pm 1.44$ (mean $\pm \mathrm{SD}$ ). The significant percentage change for roughness was $13.3 \%$ $(p<0.01)$ by paired $t$-test. Figure 6 illustrates these changes graphically.

\section{Smoothness}

Table 7 summarizes the results relating to this parameter. At baseline (D0), the mean smoothness was 41.36 AU \pm 14.02 (mean $\pm \mathrm{SD}$ ), and at D14, it was 36.01 AU士10.29 (mean \pm SD. The percentage change for smoothness was $12.9 \%$, and this

Table 4 Firmness at baseline (day 0) and end of trial (day 14)

\begin{tabular}{llllllll}
\hline \multicolumn{2}{l}{ Firmness (AU) } & & & & & & \\
\hline & Time & N & Median & Mean & SD & Min & Max \\
\hline \multirow{2}{*}{ Suvex } & OD & 32 & 0.267 & 0.264 & 0.071 & 0.150 & 0.410 \\
& I4D & 32 & 0.227 & 0.226 & 0.075 & 0.116 & 0.316 \\
Control & OD & 32 & 0.268 & 0.263 & 0.07 & 0.151 & 0.416 \\
& I4D & 32 & 0.269 & 0.262 & 0.072 & 0.153 & 0.414 \\
\hline
\end{tabular}

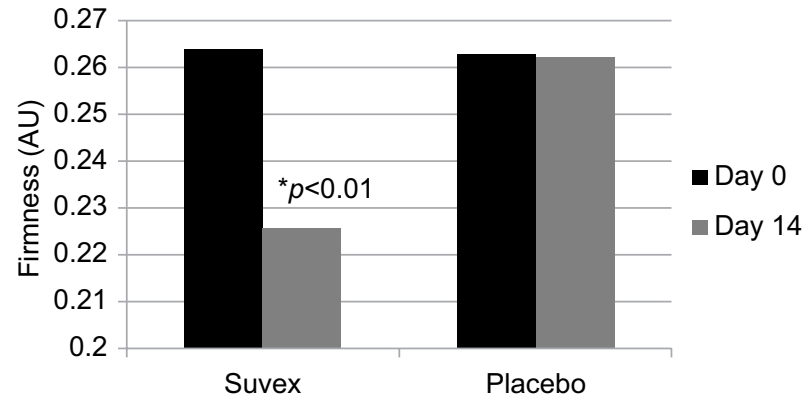

Figure 4 Mean firmness at baseline (day 0) and end of trial (day 14). 
Table 5 Erythema at baseline (day 0 ) and end of trial (day 14)

\begin{tabular}{|c|c|c|c|c|c|c|c|}
\hline \multicolumn{8}{|c|}{ Erythema (AU) } \\
\hline & Time & $\mathbf{N}$ & Median & Mean & SD & Min & Max \\
\hline \multirow[t]{2}{*}{ Suvex } & OD & 32 & 319 & 321.8 & 79.5 & 210 & 551 \\
\hline & I4D & 32 & 293.5 & 292.2 & 76.5 & 180 & 490 \\
\hline \multirow[t]{2}{*}{ Control } & OD & 32 & 317 & 321.5 & 79.4 & 211 & 550 \\
\hline & I4D & 32 & 316.2 & 320.8 & 79.8 & 210 & 553 \\
\hline
\end{tabular}

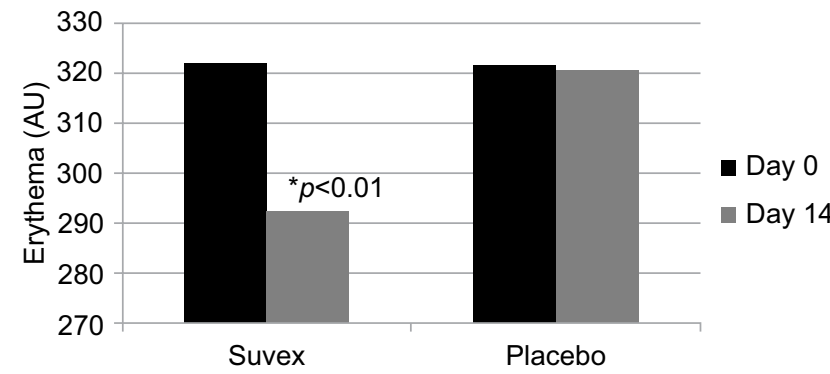

Figure 5 Erythema at baseline (day 0) and end of trial (day 14).

Table 6 Roughness at baseline (day 0) and end of trial (day 14).

\begin{tabular}{llllllll}
\hline \multicolumn{2}{l}{ Roughness (AU) } & & & & & & \\
\hline & Time & N & Median & Mean & SD & Min & Max \\
\hline \multirow{4}{*}{ Suvex } & OD & 32 & 1.85 & 2.25 & 1.31 & 0.80 & 5.88 \\
& I4D & 32 & 2.10 & 2.55 & 1.44 & 0.79 & 5.37 \\
& OD & 32 & 1.84 & 2.25 & 1.30 & 0.81 & 5.89 \\
& I4D & 32 & 1.85 & 2.25 & 1.31 & 0.81 & 5.88 \\
\hline
\end{tabular}

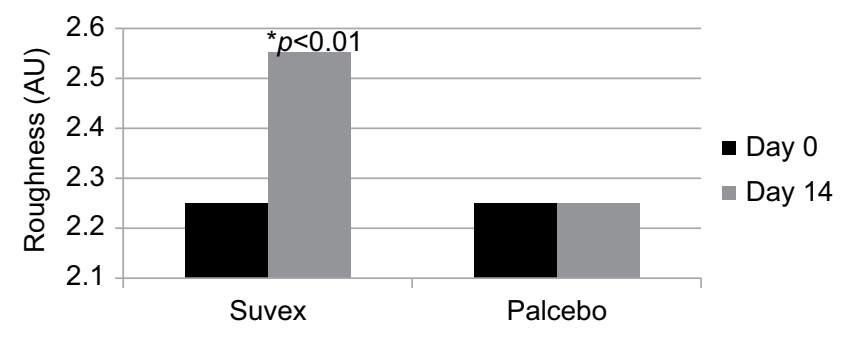

Figure 6 Mean roughness at baseline (day 0 ) and end of trial (day 14).

was statistically significant $(p<0.01)$ by paired $t$-test. Figure 7 illustrates these changes graphically.

\section{Product satisfaction scores}

Twenty participants completed the product satisfaction scores. The reason given by all twelve participants who failed to complete this element of the investigation was lack of time. The results for Suvex generated high scores for user satisfaction for most criteria. The level of satisfaction that the product delivered as an emollient was a score of 4.1 out of a possible maximum score of 5 , its effectiveness as an emollient was a score of 3.8, how well it was absorbed into the skin was 4.4, and its efficacy was 3.9. Sixty-six percent of participants said they would change their current
Table 7 Smoothness at baseline (day 0) and end of trial (day 14)

\begin{tabular}{|c|c|c|c|c|c|c|c|}
\hline \multicolumn{8}{|c|}{ Smoothness (AU) } \\
\hline & Time & $\mathbf{N}$ & Median & Mean & SD & Min & Max \\
\hline \multirow[t]{2}{*}{ Suvex } & OD & 32 & 38.21 & 41.36 & 14.02 & 24.59 & 87.70 \\
\hline & $14 \mathrm{D}$ & 32 & 32.56 & 36.01 & 10.29 & 20.51 & 69.43 \\
\hline \multirow[t]{2}{*}{ Control } & OD & 32 & 38.23 & 41.39 & 14.03 & 24.60 & 87.73 \\
\hline & I4D & 32 & 38.21 & 41.35 & 14.04 & 24.58 & 87.71 \\
\hline
\end{tabular}

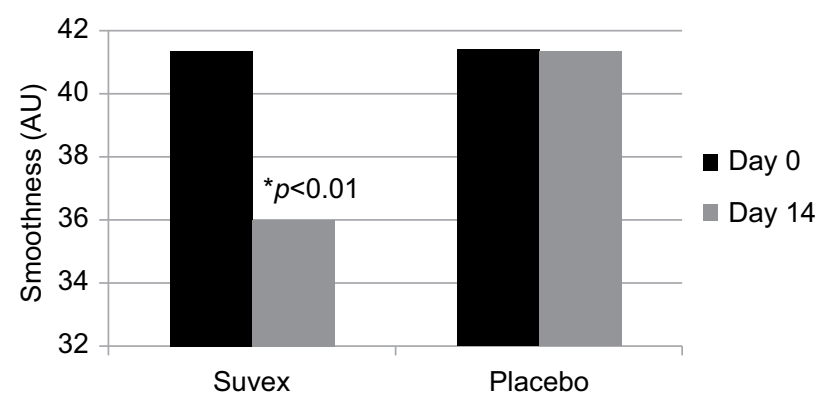

Figure 7 Mean smoothness at baseline (day 0) and end of trial (day 14).

emollient to Suvex, 90\% said they would recommend the product to others, while $77 \%$ said they would ask their health professional to prescribe Suvex should it become available on prescription. Only six negative comments are related to the odour of the product. As the natural product odour has not been neutralized and is naturally fragrance free, this observation is not unexpected.

\section{Discussion}

Primarily this study aimed to objectively assess the effect of Suvex on the parameters of skin function over 14 days of use. The regimen of twice daily application was chosen to replicate the recommendations for frequent application of an emollient. Suvex delivered a highly statistically significant improvement in reducing TEWL, delivering increases in the moisture content, the smoothness, the elasticity, and the firmness of the skin. It also reduced erythema and skin roughness. These benefits demonstrate that Suvex is an effective emollient that is able to moisturize the skin and deliver improvements in skin function that will be beneficial to patients with dry skin conditions such as eczema. Because no prior data relating to the emollient and objective measures of skin function exist, it was deemed inappropriate to use the product directly on the skin affected by eczema. However, although, in this instance, the product was not assessed on that parameter, inclusion criteria did require that participants had a history of the condition. Given that the barrier function of the skin these individuals is not only impaired in the symptomatic areas, but also in the parts of the skin that appear to have a normal appearance, based upon these results it now 
seems appropriate to assess Suvex in the frank condition where emollient therapy might be appropriate.

Clearly, the most important primary consideration relating to an emollient is efficacy. However, because patients have to use these products often on a daily basis for many years, there needs to be a high level of satisfaction with their physical characteristics and acceptance of use to ensure continued application. Here, participants rated the product as highly satisfactory in terms of ease of absorption into the skin. They reported satisfaction with it as an emollient (both characteristics scored $>4$ ). However, from a therapeutic perspective, the most relevant fact was the number of those reporting that they would use it again, when compared with their existing emollient (66\%). Another preference evaluation reported by other investigators unsurprisingly found ointments to be the patients' least favorite type of emollient. Furthermore, $81 \%$ of those participating in that study reported that the emollient they most preferred to use was not the one that was currently prescribed by their healthcare practitioner.

Most healthcare practitioners prefer emollients that appear gentle to the skin without issues around sensitivity, especially for patients with atopic eczema. ${ }^{30}$ However, many emollients contain ingredients such as the preservative phenoxyethanol and emulsifiers such as sodium lauryl sulfate that are likely to sensitize the skin in susceptible individuals. ${ }^{31,32}$ This is particularly the case with aqueous cream formulations that contain both ingredients and are likely to cause discomfort and stinging when left on the skin to deliver emollient effects in these subjects. Undesirable effects at a cellular level with increased TEWL and inflammatory protease activity occurring along with reduced thickness of the stratum corneum have been reported ${ }^{33}$ ). However, throughout the 14 days of use of Suvex, no adverse symptoms or signs were reported.

The combination of objective measures of skin function, together with subjective assessments of acceptability and performance under conditions similar to those likely to occur in normal clinical usage, demonstrates the therapeutic suitability of the product as an emollient for those with dry and eczematous skin. The outcomes observed in this investigation also demonstrate that Suvex delivers a similar emollient effect to other commercially available products currently available for prescription in the UK by clinicians ${ }^{34}$

Because in reality emollients are likely to be employed both for a long duration and in varying quantities and frequencies, possible limitations of the approach taken in this study are the relatively short treatment time of 14 days and the regimen of twice daily application. Researchers have identified that healthcare professionals continue to be an important resource regarding recommendations relating to emollient usage. The majority believe that an ideal emollient is a transparent or white cream without fragrance that needs to be used no more than two to three times per day. If the emollient meets the preferences of patients or parents selecting an emollient for their children, then compliance appears to be enhanced. Given that the positive outcomes of this open-label pilot study appear to meet these criteria, the next stage will be to undertake a double-blind comparative study in patients with frank atopic eczema.

\section{Conclusion}

Suvex has been demonstrated in this pilot study to improve the most important objective measures of skin function in those prone to eczema. The regimen of 2-week application, twice daily, reduced TEWL by $12 \%$; increased hydration by $16.7 \%$; enhanced elasticity and firmness by $13.5 \%$ and $14.3 \%$, respectively; reduced erythema by $10.1 \%$; reduced roughness by $13.3 \%$, and improved smoothness by $12.9 \%$ in the healthy skin of a group of individuals prone to eczema. The study results show that Suvex soothes, hydrates, and repairs dry, red, and eczema-prone skin.

\section{Disclosure}

The author reports no conflicts of interest in this work.

\section{References}

1. Cork MJ. The importance of skin barrier function. J Dermatolog Treat. 1997;(Supp1 8):S7-S13.

2. Tree S, Marks S. An explanation for the "placebo" effect of bland ointment bases. Br J Dermatol. 1975;92(2):195-198.

3. Comaish S, Lennart J. Site of action of methotrexate in psoriasis. Arch Dermatol. 1969;100(1):99-105.

4. Lewis-Jones S, Mugglestone MA. Management of atopic eczema in children aged up to 12 years: summary of NICE guidance. BMJ. 2007;335(7632):1263-1264.

5. Lodén M. The skin barrier and use of moisturizers in atopic dermatitis Clin Dermatol. 2003;21(2):145-157.

6. Watkins P. Using emollients to restore and maintain skin integrity. Nurs Stand. 2008;22(41):51-57.

7. Rawlings $\mathrm{AV}$, Harding CR. Moisturization and skin barrier function. Dermatol Ther. 2004;17(Suppl 1):43-48.

8. Kashibuchi N, Hirai Y, O’Goshi K, Tagami H. Three-dimensional analyses of individual corneocytes with atomic force microscope morphological changes related to age, location and to the pathologic skin conditions. Skin Res Technol. 2002;8(4):203-211.

9. Voegeli D. The role of emollients in the care of patients with dry skin. Nurs Stand. 2007;22(7):62-68.

10. Moncrieff G, Cork M, Lawton S, Kokiet S, Daly C, Clark C. Use of emollients in dry-skin conditions: consensus statement. Clin Exp Dermatol. 2013;38(3):231-238.

11. Sidbury R, Poorsattar S. Pediatric atopic dermatitis: should we treat it differently? Dermatol Ther. 2006;19(2):83-90.

12. Clark C. How to choose a suitable emollient. Pharm J. 2004;7:4-19.

13. Elias PM, Steinhoff M. "Outside-to-inside" (and now back to "outside") pathogenic mechanisms in atopic dermatitis. J Invest Dermatol. 2008;128(5):1067-1070. 
14. Imokawa G, Abe A, Jin K, Higaki Y, Kawashima M, Hidano A. Decreased level of ceramides in stratum corneum of atopic dermatitis: an etiologic factor in atopic dry skin? J Invest Dermatol. 1991;96(4):523-526.

15. Di Nardo A, Wertz P, Giannetti A, Seidenari S. Ceramide and cholesterol composition of the skin of patients with atopic dermatitis. Acta Dermato-Venereologica. 1998;78(1):27-30.

16. Yamamoto A, Serizawa S, Ito M, Sato Y. Stratum corneum lipid abnormalities in atopic dermatitis Arch Dermatol Res.1991;283(4):219-223.

17. Jungersted JM, Scheer H, Mempel M, et al. Stratum corneuml lipids, skin barrier function and filaggrin mutations in patients with atopic eczema. Allergy. 2010;65(7):911-918.

18. Janssens M, van Smeden J, Gooris GS, et al. Lamellar lipid organization and ceramide composition in the stratum corneum of patients with atopic eczema. J Invest Dermatol. 2011;131(10):2136-2138.

19. Pilgram G, Vissers J, van der Meulen H, et al. Aberrant lipid organization in stratum corneum of patients with atopic dermatitis and lamellar ichthyosis. J Invest Dermatol. 2001;117(3):710-717.

20. Ong PY. Emerging drugs for atopic dermatitis. Expert Opin Emerg Drugs. 2009:14(1):165-179.

21. Chase EP, Armstrong AW. Advances in management of atopic dermatitis: new therapies and novel uses of existing treatments. Semin Cutan Med Surg. 2012;31(1):17-24.

22. Loden M. The clinical benefit of moisturizers. J Eur Acad Dermatol Venereol. 2005;19(6):672-688.

23. Werner Y. The water content of the stratum corneum in patients with atopic dermatitis. Acta Derm Veberol. 1986;66(4):281-284.

24. Holm EA, Wulf HC, Thomassen L, Jemec GB. Instrumental assessment of atopic eczema: validation of transepidermal water loss, stratum corneum hydration, erythema, scaling, and edema. J Am Acad Dermatol. 2006;55(5):772-780.
25. Loden, M., Hagforsen E, Lindberg M. The presence of body hair influences the measurement of skin hydration with the Corneometer. Acta Derm Venereol. 1995:75(6):449-450.

26. Almalty AM, Hamed SH, Al-Dabbak FM, Shallan AE. Short-term and long-term effects of electrical stimulation on skin properties. Physiother Res Int. 2013;18(3):157-166.

27. Clarys P, Alewaeters K, Lambrecht R, Barel AO. Skin color measurements: comparison between three instruments: the Chromameter ${ }^{\circledR}$, the DermaSpectrometer ${ }^{\circledR}$ and the Mexameter ${ }^{\circledR}$. Skin Res Technol. 2000;6(4):230-238.

28. De Paepe K, Houben E, Adam R, Wiesemann F, Rogiers V. Validation of the VapoMeter, a closed unventilated chamber system to assess transepidermal water loss vs. the open chamber Tewameter ${ }^{\mathbb{B}}$. Skin Res Technol. 2005;11(1):61-69.

29. Khan, Barkat Ali, et al. Visio Scan VC98, Corneometer MPA 5 and Tewameter MPA 5. Afr J Pharm Pharmacol. 2012;6(3):225-227.

30. Hon KL, Wang SS, Pong NH, Leung TF. The ideal moisturizer: a survey of parental expectations and practice in childhood-onset eczema. $J$ Dermatol Treat. 2013;24(1):7-12.

31. van der Valk PG. Nater JP, Bleumink E. Skin irritancy of surfactants as assessed by water vapor loss measurements. J Invest Dermatol.1984;82(3):291-293.

32. Agner T, Serup J. Sodium lauryl sulphate for irritant patch testinga dose response study using bioengineering methods for determination of skin irritation. J Invest Dermatol. 1990;95(5):543-547.

33. Mohammed D, Matts PJ, Hadgraft J, Lane ME. Influence of aqueous cream $\mathrm{BP}$ on corneocyte size, maturity, skin protease activity, protein content and transepidermal water loss. Br J Dermatol. 2011;164(6):1304-1310.

34. Wynne A, White M, Dixon AJ, Anderson S. An effective, cosmetically acceptable, novel hydro-gel emollient for the management of dry skin conditions. J Dermatol Treatment. 2002;13(2):61-66. 


\section{Supplementary materials}

\section{Ingredient listing}

Aqua (water), Oryza sativa (rice) Bran Oil, Theobroma cacao (cocoa) Seed Butter, Butyrospermum parkii (shea) Nut Butter, Rosa canina (rosehip) Seed Oil, Octyldodecanol, Glycerin, Ascophyllum nodosum (Norwegian kelp) Plant Extract, Sorbitan Olivate, Cetearyl Olivate, Gluconolactone, Sodium Polyacrylate, Zinc PCA, Tocopherol, Sodium Benzoate, Cyamopsis tetragonoloba Gum, Plantago lanceolata (lamb's tongue) Leaf Extract, Phenoxyethanol, Echium plantagineum (purple vipor's bugloss) Seed Oil, Aloe Barbadensis (aloe vera) Leaf Juice Powder, Cardiospermum halicacabum (balloon) Plant Extract, Helianthus annuus (sunflower) Seed Oil Unsaponifiables, Potassium Sorbate.

\section{Suvex satisfaction questionnaire}

How would you rate Suvex as an emollient

Very good quite good OK quite poor very poor

How effective was it as a moisturizer

$\begin{array}{lllll}5 & 4 & 3 & 2 & 1 \\ 5 & 4 & 3 & 2 & 1 \\ 5 & 4 & 3 & 2 & 1\end{array}$

How easily did it absorb into the skin

What was it about Suvex that you particularly liked.

Was there anything you didn't like about the product.

Would you swap your current emollient for Suvex yes/no

Would you recommend Suvex as an emollient to other people yes/no

Would you ask your GP/nurse to prescribe Suvex if it was available yes/no

Figure SI Suvex questionnaire. 